PROCEEDINGS OF THE

AMERICAN MATHEMATICAL SOCIETY

Volume 129, Number 10, Pages 2973-2980

S 0002-9939(01)06019-1

Article electronically published on March 14, 2001

\title{
SOME CLASSES OF TOPOLOGICAL QUASI *-ALGEBRAS
}

\author{
F. BAGARELLO, A. INOUE, AND C. TRAPANI
}

(Communicated by David R. Larson)

\begin{abstract}
The completion $\overline{\mathcal{A}}[\tau]$ of a locally convex $*$-algebra $\mathcal{A}[\tau]$ with not jointly continuous multiplication is a $*$-vector space with partial multiplication $x y$ defined only for $x$ or $y \in \mathcal{A}_{0}$, and it is called a topological quasi *-algebra. In this paper two classes of topological quasi $*$-algebras called strict $\mathrm{CQ}^{*}$-algebras and $\mathrm{HCQ}^{*}$-algebras are studied. Roughly speaking, a strict $\mathrm{CQ}^{*}$-algebra (resp. $\mathrm{HCQ}^{*}$-algebra) is a Banach (resp. Hilbert) quasi *-algebra containing a $\mathrm{C}^{*}$-algebra endowed with another involution \# and $\mathrm{C}^{*}$ norm \|\|$_{\#}$. $\mathrm{HCQ}^{*}$-algebras are closely related to left Hilbert algebras. We shall show that a Hilbert space is a $\mathrm{HCQ}^{*}$-algebra if and only if it contains a left Hilbert algebra with unit as a dense subspace. Further, we shall give a necessary and sufficient condition under which a strict $\mathrm{CQ}^{*}$-algebra is embedded in a $\mathrm{HCQ}^{*}$-algebra.
\end{abstract}

\section{IntroduCtion}

Topological quasi $*$-algebras were first introduced by Lassner [6] for the mathematical description of some quantum physical models, and after that, they have been studied by Lassner [6, 7], Trapani [10 and Bagarello-Trapani [3, 4, etc. In this paper we shall study two classes of topological quasi $*$-algebras called strict $\mathrm{CQ}^{*}$-algebras and $\mathrm{HCQ}^{*}$-algebras from a mathematical point of view but also in the perspective of possible physical applications. Let $\mathcal{A}$ be a $*$-algebra with two involutions $*$ and $\#$ and two norms \|\| and \|\|$_{\#}$ satisfying $\left\|x^{*}\right\|=\|x\|,\|x\| \leq\|x\|_{\#}$ and $\left\|x^{\#} x\right\|_{\#}=\|x\|_{\#}^{2}$ for each $x, y \in \mathcal{A}$. Then the completion $\overline{\mathcal{A}}[\|\|]$ of $\mathcal{A}[\|\|]$ is a topological quasi $*$-algebras containing (under natural assumptions) two $\mathrm{C}^{*}$-algebras $\mathcal{A}_{\#}\left[\|\|_{\#}\right]$ and $\mathcal{A}_{b}\left[\|\|_{b}\right]$ with different involutions \# and $b$, respectively, which are connected by the isometric involution $J: x \rightarrow x^{*}$. This is called a pseudo $C Q^{*}$ algebra. If $\|x\|_{\#}=\sup \{\|x y\| ;\|y\| \leq 1\}$, then $\overline{\mathcal{A}}[\|\|]$ is a particular kind of $\mathrm{CQ}^{*}$ algebra as defined and studied in 1, 2, and it is called a strict $C Q^{*}$-algebra, and denoted by $\left(\overline{\mathcal{A}}[\|\|], \#,\|\|_{\#}\right)$. Let $\overline{\mathcal{A}}[\|\|]$ be a topological quasi $*$-algebra with isometric involution $J: x \rightarrow x^{*}$ and Hilbertian norm \|\| . If $\mathcal{A}$ has another involution \# satisfying $\|x\| \leq\left\|L_{x}\right\|$ and $L_{x}^{*}=L_{x} \#$ for each $x \in \mathcal{A}$, where $L_{x}$ is the bounded linear operator on the Hilbert space $\overline{\mathcal{A}}[\|\|]$ defined by $L_{x} y=x y, y \in \mathcal{A}$, then $\overline{\mathcal{A}}[\|\|]$ is a strict $\mathrm{CQ}^{*}$-algebra with involution \# and $\mathrm{C}^{*}$-norm $\|x\|_{\#} \equiv\left\|L_{x}\right\|, x \in \mathcal{A}$, and it is called a $H C Q^{*}$-algebra and denoted by $(\overline{\mathcal{A}}[\|\|], \#)$. HCQ ${ }^{*}$-algebras are closely related to left Hilbert algebras. Let $(\overline{\mathcal{A}}[\|\|], \#)$ be an $\mathrm{HCQ}^{*}$ algebra. Then $\mathcal{A}$ is

Received by the editors February 20, 2000.

2000 Mathematics Subject Classification. Primary 46K70.

Key words and phrases. Topological quasi *-algebras, $\mathrm{CQ}^{*}$-algebras, $\mathrm{HCQ}^{*}$-algebras.

(C)2001 American Mathematical Society 
a left Hilbert algebra in the Hilbert space $\overline{\mathcal{A}}[\|\|]$ with involution \#, and the full left Hilbert algebra $\mathcal{A}^{\prime \prime}$ of $\mathcal{A}$ has unit. But, the isometric involution $J$ does not necessarily coincide with the modular conjugation operator $J_{\mathcal{A}}$ of the left Hilbert algebra $\mathcal{A}$. If $J_{\mathcal{A}}=J$, then the $\mathrm{HCQ}^{*}$-algebra $(\overline{\mathcal{A}}[\|\|], \#)$ is said to be standard. Suppose that $(\overline{\mathcal{A}}[\|\|], \#)$ is standard. Then $\mathcal{A}$ is contained in the maximal Tomita algebra $\left(\mathcal{A}^{\prime \prime}\right)_{0}$ of $\mathcal{A}^{\prime \prime}$ and $\left(\overline{\left(\mathcal{A}^{\prime \prime}\right)_{0}}[\|\|], \#\right)$ is a standard $\mathrm{HCQ}^{*}$-algebra with the oneparameter group $\left\{\Delta_{\mathcal{A}}^{i t}\right\}_{t \in \mathbb{R}}$ of $*$-automorphisms, where $\Delta_{\mathcal{A}}$ is the modular operator of $\mathcal{A}$. From these results, it is shown that a Hilbert space is a standard $\mathrm{HCQ}^{*}$ algebra if and only if it contains a left Hilbert algebra as dense subspace. Finally, we give a necessary and sufficient condition under which a strict $\mathrm{CQ}^{*}$-algebra is embedded into a standard $\mathrm{HCQ}^{*}$-algebra using the GNS-construction of positive sesquilinear form on the strict $\mathrm{CQ}^{*}$-algebra $\overline{\mathcal{A}}[\|\|]$.

\section{Strict $\mathrm{CQ}^{*}$-ALGEBras AND $\mathrm{HCQ}^{*}$-AlgEbras}

Let $\mathcal{A}[\|\|]$ be a normed $*$-algebra with isometric involution $*$ and separately (but not jointly) continuous multiplication. Then the completion, $\overline{\mathcal{A}}[\|\|]$, of $\mathcal{A}[\|\|]$ is a topological quasi $*$-algebra that we call, as is natural, a Banach quasi $*$-algebra. In particular, if \|\| is a Hilbertian norm, then $\overline{\mathcal{A}}[\|\|]$ is called a Hilbert quasi $*$-algebra. For any $a \in \overline{\mathcal{A}}[\|\|]$ we put

$$
L_{a} x=a x \text { and } R_{a} x=x a, \quad x \in \mathcal{A} .
$$

Then $L_{a}$ and $R_{a}$ are linear maps of $\mathcal{A}$ into $\overline{\mathcal{A}}[\|\|]$. In particular, if $a \in A$, then $L_{a}$ and $R_{a}$ can be extended to bounded linear operators on the Banach space $\overline{\mathcal{A}}[\|\|]$ and they are denoted by the same symbols $L_{a}$ and $R_{a}$.

Let $\overline{\mathcal{A}}[\|\|]$ be a Banach quasi $*$-algebra and assume that the $*$-algebra $\mathcal{A}$ has another norm \|\|$_{\#}$ and another involution \# satisfying the following conditions:

(a.1) $\left\|x^{\#} x\right\|_{\#}=\|x\|_{\#}^{2}, \quad \forall x \in \mathcal{A}$.

(a.2) $\|x\| \leq\|x\|_{\#}, \quad \forall x \in \mathcal{A}$.

(a.3) $\|x y\| \leq\|x\|_{\#}\|y\|, \quad \forall x, y \in \mathcal{A}$.

Then by (a.2), the identity map $i: \mathcal{A}\left[\|\|_{\#}\right] \rightarrow \mathcal{A}[\|\|]$ has a continuous extension $\hat{i}$ from the completion $\mathcal{A}_{\#}$ of $\mathcal{A}\left[\|\|_{\#}\right]$ ( $\mathcal{A}_{\#}$ is, of course, a $\mathrm{C}^{*}$-algebra) into $\overline{\mathcal{A}}[\|\|]$. If $\hat{i}$ is injective, then $\mathcal{A}_{\#}$ is (identified with) a dense subspace of $\overline{\mathcal{A}}$. This happens if, and only if,

(a.4) two norms \|\| and \|\|$_{\#}$ are compatible in the following sense [5]: for any sequence $\left\{x_{n}\right\} \subset \mathcal{A}$ such that $\left\|x_{n}\right\| \rightarrow 0$ and $x_{n} \rightarrow x$ in $\mathcal{A}_{\#}\left[\|\|_{\#}\right], x=0$ results, i.e. if $\hat{i}^{-1}: \mathcal{A}[\|\|] \rightarrow \mathcal{A}_{\#}\left[\|\|_{\#}\right]$ is closable.

Definition 2.1. A Banach quasi $*$-algebra $\overline{\mathcal{A}}[\|\|]$ is said to be a psuedo $C Q^{*}$-algebra if the $*$-algebra $\mathcal{A}$ has a another norm \|\|$_{\#}$ and another involution \# satisfying the conditions (a.1)-(a.4) above. Furthermore, if $\|x\|_{\#}=\left\|L_{x}\right\| \equiv \sup \{\|x y\| ; y \in$ $\mathcal{A}$ s.t. $\|y\| \leq 1\}$ for each $x \in \mathcal{A}$, then $\overline{\mathcal{A}}[\|\|]$ is said to be a strict $C Q^{*}$-algebra.

A pseudo $\mathrm{CQ}^{*}$-algebra $\overline{\mathcal{A}}[\|\|]$ is fully determined by the involution \# and the $\mathrm{C}^{*}$-norm \|\|$_{\#}$, and so it will often be denoted by $\left(\overline{\mathcal{A}}[\|\|], \#,\|\|_{\#}\right)$. On the other hand, a strict $\mathrm{CQ}^{*}$-algebra is fully determined when the new involution \# is known; so it can be simply denoted as $(\overline{\mathcal{A}}[\|\|]$, \#), making lighter in this way the notation introduced by two of us in [1, 2]. Let $\left(\overline{\mathcal{A}}[\|\|], \#,\|\|_{\#}\right)$ be a pseudo $\mathrm{CQ}^{*}$-algebra and, as above, let $\mathcal{A}_{\#}$ be the $\mathrm{C}^{*}$-algebra obtained by completing the \#-algebra $\mathcal{A}$ with respect to the $\mathrm{C}^{*}$-norm \|\|$_{\#}$. Let $J$ be the involution $*$ of the Banach quasi 
*-algebra $\overline{\mathcal{A}}[\|\|]$. Then $\mathcal{A}_{b} \equiv J \mathcal{A}_{\#}$ is a $\mathrm{C}^{*}$-algebra equipped with the operations $x^{*}+y^{*} \equiv(x+y)^{*}, \lambda x^{*} \equiv(\bar{\lambda} x)^{*}, x^{*} y^{*} \equiv(y x)^{*}$, the involution $\left(x^{*}\right)^{b} \equiv x^{\# *}$ and the $\mathrm{C}^{*}$-norm $\left\|x^{*}\right\|_{b} \equiv\|x\|_{\#}, \forall x, y \in \mathcal{A}_{b}$.

Proposition 2.2. A pseudo $C Q^{*}$-algebra $\left(\overline{\mathcal{A}}[\|\|], \#,\|\|_{\#}\right)$ contains two $C^{*}$-algebras $\mathcal{A}_{\#}$ and $\mathcal{A}_{b} \equiv J \mathcal{A}_{\#}$ with different involutions \# and $b$, respectively, as dense subalgebra. In particular, if $\left(\overline{\mathcal{A}}[\|\|], \#,\|\|_{\#}\right)$ is a strict $C Q^{*}$-algebra, then $L_{\mathcal{A}_{\#}}$ and $R_{\mathcal{A}_{b}}$ are $C^{*}$-algebras, $L_{x} R_{y}=R_{y} L_{x}$ for each $x \in \mathcal{A}_{\#}$ and $y \in \mathcal{A}_{b}$ and $R_{\mathcal{A}_{b}}=J L_{\mathcal{A}_{\#}} J$.

By Proposition 2.2, every strict $\mathrm{CQ}^{*}$-algebra is a $\mathrm{CQ}^{*}$-algebra in the sense of 1, 2] but the converse is not true in general $\left(\left(\mathcal{A}_{\#} \cap \mathcal{A}_{b}\right)\right.$ is not required to be \#-invariant).

We summarize the situation with the following scheme:

$$
\begin{aligned}
& \begin{array}{cccc}
\mathcal{A}_{\#} & \subset & \overline{\mathcal{A}}[\|\|]
\end{array} \\
& \text { normed *-algebra } \quad \mathrm{C}^{*} \text {-algebras } \quad \mathrm{CQ}^{*} \text {-algebra, }
\end{aligned}
$$

which summarizes the situation: the *-algebra $\mathcal{A}[\|\|]$ is contained in its closures, $\mathcal{A}_{\#}=\overline{\mathcal{A}}\left[\|\|_{\#}\right]$ and $\mathcal{A}_{b}=\overline{\mathcal{A}}\left[\|\|_{b}\right]=J \mathcal{A}_{\#}$. These $\mathrm{C}^{*}$-algebras, moreover, are both contained in $\overline{\mathcal{A}}[\|\|]$.

Definition 2.3. A Hilbert quasi $*$-algebra $\overline{\mathcal{A}}[\|\|]$ is said to be a $H C Q^{*}$-algebra if there is another involution \# of $\mathcal{A}$ such that $L_{x}^{*}=L_{x} \#$ and $\|x\| \leq\left\|L_{x}\right\|$ for each $x \in \mathcal{A}$. Here we denote it by $(\overline{\mathcal{A}}[\|\|], \#)$.

$\mathrm{HCQ}^{*}$-algebras are closely related to left Hilbert algebras. Before going forth, for the reader's convenience, we briefly review the definitions and the basic properties of left Hilbert algebras. A $*$-algebra $\mathfrak{A}$ with involution \# is said to be a left Hilbert algebra if it is a dense subspace in a Hilbert space $\mathcal{H}$ with inner product $(\mid)$ satisfying the following conditions:

(i) For any $x \in \mathfrak{A}$ the map $y \in \mathfrak{A} \rightarrow x y \in \mathfrak{A}$ is continuous.

(ii) $(x y \mid z)=\left(y \mid x^{\#} z\right), \forall x, y, z \in \mathfrak{A}$.

(iii) $\mathfrak{A}^{2} \equiv\{x y ; x, y \in \mathcal{A}\}$ is total in $\mathcal{H}$.

(iv) The involution $x \rightarrow x^{\#}$ is closable in $\mathcal{H}$.

By (i), for any $x \in \mathfrak{A}$ we denote by $\pi_{\mathfrak{A}}(x)$ the unique continuous linear extension to $\mathcal{H}$ of the map $y \in \mathfrak{A} \rightarrow x y \in \mathfrak{A}$; then $\pi_{\mathfrak{A}}$ is a $*$-representation of $\mathfrak{A}$ on $\mathcal{H}$. We denote by $S_{\mathfrak{A}}$ the closure of the involution \#. Let $S_{\mathfrak{A}}=J_{\mathfrak{A}} \Delta_{\mathfrak{A}}^{\frac{1}{2}}$ be the polar decomposition of $S_{\mathfrak{A}}$. Then $J_{\mathfrak{A}}$ is an isometric involution on $\mathcal{H}$ and $\Delta_{\mathfrak{A}}$ is a nonsingular positive self-adjoint operator in $\mathcal{H}$ such that $S_{\mathfrak{A}}=J_{\mathfrak{A}} \Delta_{\mathfrak{A}}^{\frac{1}{2}}=\Delta_{\mathfrak{A}}^{-\frac{1}{2}} J_{\mathfrak{A}}$ and $S_{\mathfrak{A}}^{*}=J_{\mathfrak{A}} \Delta_{\mathfrak{A}}^{-\frac{1}{2}}=\Delta_{\mathfrak{A}}^{\frac{1}{2}} J_{\mathfrak{A}}$, and $J_{\mathfrak{A}}$ is called the modular conjugation operator of $\mathfrak{A}$ and $\Delta_{\mathfrak{A}}$ is called the modular operator of $\mathfrak{A}$. We define the commutant $\mathfrak{A}^{\prime}$ of $\mathfrak{A}$ as follows: For any $y \in \mathcal{D}\left(S_{\mathfrak{A}}^{*}\right)$ we put $\pi_{\mathfrak{A}}^{\prime}(y) x=\pi_{\mathfrak{A}}(x) y, x \in \mathfrak{A}$ and put $\mathfrak{A}^{\prime}=\{y \in$ $\mathcal{D}\left(S_{\mathfrak{A}}^{*}\right) ; \pi_{\mathfrak{A}}^{\prime}(y)$ is bounded\}. Then $\mathfrak{A}^{\prime}$ is a left Hilbert algebra in $\mathcal{H}$ with involution $S_{\mathfrak{A}}^{*}$ and multiplication $y_{1} y_{2} \equiv \pi_{\mathfrak{A}}^{\prime}\left(y_{2}\right) y_{1}$. Similarly, the commutant $\mathfrak{A}^{\prime \prime}$ of $\mathfrak{A}^{\prime}$ is defined by $\mathfrak{A}^{\prime \prime}=\left\{x \in \mathcal{D}\left(S_{\mathfrak{A}}\right) ; y \in \mathfrak{A}^{\prime} \rightarrow x y\right.$ is continuous $\}$. For any $x \in \mathfrak{A}^{\prime \prime}$ we denote by $\pi_{\mathfrak{A}}(x)$ the unique continuous linear operator on $\mathcal{H}$ such that $\pi_{\mathfrak{A}}(x) y=$ $\pi_{\mathfrak{A}}^{\prime}(y) x, y \in \mathfrak{A}^{\prime}$. Then $\mathfrak{A}^{\prime \prime}$ is a left Hilbert algebra in $\mathcal{H}$ with involution $S_{\mathfrak{A}}$ and multiplication $x_{1} x_{2} \equiv \pi_{\mathfrak{A}}\left(x_{1}\right) x_{2}$ containing $\mathfrak{A}$. A left Hilbert algebra $\mathfrak{A}$ is said to be full if $\mathfrak{A}=\mathfrak{A}^{\prime \prime}$. It is well-known as the Tomita fundamental theorem that 
$J_{\mathfrak{A}} \pi_{\mathfrak{A}}(\mathfrak{A})^{\prime \prime} J_{\mathfrak{A}}=\pi_{\mathfrak{A}}(\mathfrak{A})^{\prime}$ and $\Delta_{\mathfrak{A}}^{i t} \pi_{\mathfrak{A}}(\mathfrak{A})^{\prime \prime} \Delta_{\mathfrak{A}}^{-i t}=\pi_{\mathfrak{A}}(\mathfrak{A})^{\prime \prime}, \forall t \in \mathbb{R}$. Let $\mathfrak{A}$ be a full left Hilbert algebra in $\mathcal{H}$, and $\mathfrak{A}_{0} \equiv\left\{x \in \bigcap_{\alpha \in \mathbb{C}} \mathcal{D}\left(\Delta_{\mathfrak{A}}^{\alpha}\right) ; \Delta_{\mathfrak{A}}^{\alpha} x \in \mathfrak{A}, \forall \alpha \in \mathbb{C}\right\}$. Then $\mathfrak{A}_{0}$ is a left Hilbert subalgebra in $\mathcal{H}$ such that $\mathfrak{A}_{0}^{\prime \prime}=\mathfrak{A}, J_{\mathfrak{A}} \mathfrak{A}_{0}=\mathfrak{A}_{0}$ and $\left\{\Delta_{\mathfrak{A}}^{\alpha} ; \alpha \in \mathbb{C}\right\}$ is a complex one-parameter group of automorphisms of $\mathfrak{A}_{0} \operatorname{such}$ that $\left(\Delta_{\mathfrak{A}}^{\alpha} x\right)^{\#}=\Delta_{\mathfrak{A}}^{-\bar{\alpha}} x^{\#}$ and $\left(\Delta_{\mathfrak{A}}^{\alpha} x\right)^{*}=\Delta_{\mathfrak{A}}^{-\bar{\alpha}} x^{*}$ for each $\alpha \in \mathbb{C}$ and $x \in \mathfrak{A}_{0}$. This $\mathfrak{A}_{0}$ is called the maximal Tomita algebra of $\mathfrak{A}$. For more details, see [8, 9, 11].

Proposition 2.4. Suppose that $(\overline{\mathcal{A}}[\|\|], \#)$ is a $H C Q^{*}$-algebra. Then the following statements hold:

(i) $(\overline{\mathcal{A}}[\|\|], \#)$ is a strict $C Q^{*}$-algebra with the $C^{*}$-norm $\|x\|_{\#}=\left\|L_{x}\right\|, x \in \mathcal{A}$.

(ii) $\mathcal{A}$ is a left Hilbert algebra in the Hilbert space $\mathcal{H} \equiv \overline{\mathcal{A}}[\|\|]$ whose full left Hilbert algebra $\mathcal{A}^{\prime \prime}$ has a unit $u$.

Proof. (i) The proof is mostly trivial. We prove only that the condition (a.4) is satisfied in this case. Indeed, if $\left\{x_{n}\right\} \subset \mathcal{A}$ is a sequence such that $\left\|x_{n}\right\| \rightarrow 0$ and $x_{n} \rightarrow x$ in $\mathcal{A}_{\#}\left[\|\|_{\#}\right]$, then by the assumption $L_{x_{n}} \rightarrow L_{x}$ with respect to the operator norm. The continuity of the multiplication in $\mathcal{A}[\|\|]$ easily implies that $L_{x}=0$; thus $\|x\|_{\#}=0$ and $x=0$.

(ii) We first show that $\mathcal{A}$ is a left Hilbert algebra in $\mathcal{H}$ with involution \#. Since the $\mathrm{C}^{*}$-algebra $\mathcal{A}_{\#}$ has an approximate identity $\left\{u_{\alpha}\right\}, \mathcal{A}$ is dense in the $\mathrm{C}^{*}$-algebra $\mathcal{A}_{\#}$ and $\|x\| \leq\|x\|_{\#}$ for each $x \in \mathcal{A}$, and then it follows that $\mathcal{A}^{2}$ is total in $\mathcal{A}[\|\|]$. The assumption $L_{x}^{*}=L_{x \#}(\forall x \in \mathcal{A})$ implies that $(x y \mid z)=\left(y \mid x^{\#} z\right)$ for each $x, y, z \in \mathcal{A}$, where $(\mid)$ is the inner product defined by the Hilbertian norm \|\| . Further, we have $\pi_{\mathcal{A}}(x)=L_{x}, \forall x \in \mathcal{A}$ and $\pi_{\mathcal{A}}(x)$ is bounded. Take any sequence $\left\{x_{n}\right\}$ in $\mathcal{A}$ such that $\lim _{n \rightarrow \infty}\left\|x_{n}\right\|=0$ and $\lim _{n \rightarrow \infty}\left\|x_{n} \#-y\right\|=0$. Then it follows that $\left(y \mid x_{1} x_{2}^{b}\right)=\lim _{n \rightarrow \infty}\left(x_{n}^{\#} \mid x_{1} x_{2}^{b}\right)=\lim _{n \rightarrow \infty}\left(x_{2} x_{1}^{b} \mid x_{n}\right)=0$ for each $x_{1}, x_{2} \in \mathcal{A}$, which implies that $x \in \mathcal{A} \mapsto x^{\#} \in \mathcal{A}$ is closable. Thus $\mathcal{A}$ is a left Hilbert algebra in $\mathcal{H}$ with the involution \#. We next show that the full left Hilbert algebra $\mathcal{A}^{\prime \prime}$ has a unit $u$. For any $\varepsilon>0$ and for any finite subsets $\left\{x_{1}, \ldots, x_{m}\right\}$ and $\left\{y_{1}, \ldots, y_{m}\right\}$ of $\mathcal{A}$, we define the set

$$
\begin{aligned}
& K\left(\varepsilon ;\left\{x_{1}, \ldots, x_{m}\right\},\left\{y_{1}, \ldots, y_{m}\right\}\right) \\
& \quad=\left\{a \in \mathcal{H} ;\|a\| \leq 1,\left|\left(a x_{k}-x_{k} \mid y_{k}\right)\right| \leq \varepsilon\right. \\
& \left.\quad \text { and }\left|\left(x_{k} a-x_{k} \mid y_{k}\right)\right| \leq \varepsilon, k=1, \ldots, m\right\} .
\end{aligned}
$$

Since the $\mathrm{C}^{*}$-algebra $\mathcal{A}_{\#}$ has an approximate identity and $\|x\| \leq\|x\|_{\#}$ for each $x \in \mathcal{A}_{\#}$, it follows that $K\left(\varepsilon ;\left\{x_{1}, \ldots, x_{m}\right\},\left\{y_{1}, \ldots, y_{m}\right\}\right) \neq \phi$. Now let $\mathcal{K}$ be the family of all subsets $K\left(\varepsilon ;\left\{x_{1}, \ldots, x_{m}\right\},\left\{y_{1}, \ldots, y_{m}\right\}\right)$ where $\varepsilon>0$ and $\left\{x_{1}, \ldots, x_{m}\right\}$, $\left\{y_{1}, \ldots, y_{m}\right\}$ are finite subsets. Then $\mathcal{K}$ is a family of non-empty weakly closed subsets of the weakly compact set $\mathcal{H}_{1} \equiv\{a \in \mathcal{H} ;\|a\| \leq 1\}$. Hence, the intersection of all the sets in $\mathcal{K}$ is non-empty. Hence, an element $u$ of this intersection is such that $u$ is a quasi-unit of the topological quasi $*$-algebra $\overline{\mathcal{A}}[\|\|]$, that is, $u \in \overline{\mathcal{A}}[\|\|]$ and $u x=x u=x$ for each $x \in \mathcal{A}$. Since

$$
\left(S_{\mathcal{A}} x \mid u\right)=\left(x^{\#} \mid u\right)=\left(u \mid L_{x} u\right)=(u \mid x)
$$

for each $x \in \mathcal{A}$, it follows that $u \in \mathcal{D}\left(S_{\mathcal{A}}^{*}\right)$ and $\pi_{\mathcal{A}}^{\prime}(u)=I$. Hence, $u \in \mathcal{A}^{\prime}$ and $S_{\mathcal{A}}^{*} u=u$, which implies that

$$
\left(S_{\mathcal{A}}^{*} y \mid u\right)=\left(\pi_{\mathcal{A}}^{\prime}\left(S_{\mathcal{A}}^{*} y\right) u \mid u\right)=\left(u \mid \pi_{\mathcal{A}}^{\prime}(y) u\right)=(u \mid y)
$$


for each $y \in \mathcal{A}^{\prime}$. Hence, we have $u \in \mathcal{A}^{\prime \prime}$ and $S_{\mathcal{A}} u=u$. This completes the proof.

By Proposition 2.4, the situation of $\mathrm{HCQ}^{*}$-algebras can be schematized with the following diagram:

$$
\begin{aligned}
& \subset \mathcal{A}_{\#} \subset \mathcal{A}^{\prime \prime}=L_{\mathcal{A}}^{\prime \prime} u \quad \subset \mathcal{D}\left(S_{\mathcal{A}}\right) \subset \\
& \mathcal{A} \quad \uparrow J \quad \uparrow J_{\mathcal{A}} \quad \uparrow J_{\mathcal{A}} \quad \overline{\mathcal{A}}[\|\|] \\
& \subset \mathcal{A}_{b} \subset \mathcal{A}^{\prime}=L_{\mathcal{A}^{\prime}} u \quad \subset \mathcal{D}\left(S_{\mathcal{A}}^{*}\right) \subset
\end{aligned}
$$

We now look for conditions under which $J=J_{\mathcal{A}}$.

Lemma 2.5. Let $(\overline{\mathcal{A}}[\|\|], \#)$ be a $H C Q^{*}$-algebra. Then the following statements are equivalent:

(i) $J=J_{\mathcal{A}}$.

(ii) $\left(x^{\#} \mid x^{*}\right) \geq 0$ for each $x \in \mathcal{A}$.

Proof. (i) $\Rightarrow$ (ii) This follows from

$$
\left(x^{\#} \mid x^{*}\right)=\left(J_{\mathcal{A}} \Delta_{\mathcal{A}}^{\frac{1}{2}} x \mid J_{\mathcal{A}} x\right)=\left(x \mid \Delta_{\mathcal{A}}^{\frac{1}{2}} x\right) \geq 0, \forall x \in \mathcal{A} .
$$

(ii) $\Rightarrow$ (i) By the assumption (ii) we have $S_{\mathcal{A}}=J\left(J J_{\mathcal{A}} \Delta_{\mathcal{A}}^{\frac{1}{2}}\right)$ and $J J_{\mathcal{A}} \Delta_{\mathcal{A}}^{\frac{1}{2}} \geq 0$. The uniqueness of the polar decomposition of $S_{\mathcal{A}}$ implies $J=J_{\mathcal{A}}$.

If any one of the two equivalent statements of Lemma 2.5 holds, we say that the $\mathrm{HCQ}^{*}$-algebra $(\overline{\mathcal{A}}[\|\|], \#)$ is standard.

Remark 2.6. Let $(\overline{\mathcal{A}}[\|\|], \#)$ be a $\mathrm{HCQ}^{*}$-algebra. If it is standard, then $R_{\mathcal{A}}^{\prime}=L_{\mathcal{A}}^{\prime \prime}$. Conversely, if $R_{\mathcal{A}}^{\prime}=L_{\mathcal{A}}^{\prime \prime}$, then $J J_{\mathcal{A}}=J_{A} J$, but we don't know whether $J=J_{\mathcal{A}}$.

Since two HCQ*-algebras $(\overline{\mathcal{A}}[\|\|]$, \#) with $(\overline{\mathcal{B}}[\|\|], \#), \overline{\mathcal{A}}[\|\|]=\overline{\mathcal{B}}[\|\|]$ as Hilbert spaces, need not coincide as $\mathrm{HCQ}^{*}$-algebras, we introduce the following notion:

Definition 2.7. A $\mathrm{HCQ}^{*}$-algebra $\overline{\mathcal{A}}[\|\|]$ is said to be an extension of a $\mathrm{HCQ}^{*}$ algebra $\overline{\mathcal{B}}[\|\|]$ if $\mathcal{B}$ is a dense $*$-subalgebra of $\mathcal{A}$ and $S_{\mathcal{A}}=S_{\mathcal{B}}$.

Proposition 2.8. Let $(\overline{\mathcal{A}}[\|\|], \#)$ be a standard $H C Q^{*}$-algebra, and $\mathcal{B} \equiv\left(\mathcal{A}^{\prime \prime}\right)_{0}$ the maximal Tomita algebra of the full left Hilbert algebra $\mathcal{A}^{\prime \prime}$. Then $\left(\overline{\mathcal{B}}[\|\|], S_{\mathcal{A}}\right)$ is a standard $H C Q^{*}$-algebra and it is an extension of $\left(\overline{\mathcal{A}}[\|\|], S_{\mathcal{A}}\right)$. Further, $\left\{\Delta_{\mathcal{A}}^{i t}\right\}_{t \in \mathbb{R}}$ is a one-parameter group of $*$-automorphisms of the Hilbert quasi $*$-algebra $\tilde{\mathcal{B}}[\|\|]$, that is, $\Delta_{\mathfrak{A}}^{i t} \mathcal{B}=\mathcal{B},\left(\Delta_{\mathcal{A}}^{i t} a\right)^{*}=\Delta_{\mathcal{A}}^{i t} a^{*}, \Delta_{\mathcal{A}}^{i t}(a x)=\left(\Delta_{\mathcal{A}}^{i t} a\right)\left(\Delta_{\mathcal{A}}^{i t} x\right)$ and $\Delta_{\mathcal{A}}^{i t}(x a)=$ $\left(\Delta_{\mathcal{A}}^{i t} x\right)\left(\Delta_{\mathcal{A}}^{i t} a\right)$ for all $a \in \overline{\mathcal{B}}[\|\|], x \in \mathcal{B}$ and $t \in \mathbb{R}$.

Proof. It is almost clear that $\overline{\mathcal{B}}(\|\|)$ is a Hilbert quasi *-algebra with the involution $J_{\mathcal{A}}=J_{\mathcal{B}}$ and further $\left(\overline{\mathcal{B}}[\|\|], S_{\mathcal{A}}\right)$ is a standard $\mathrm{HCQ}^{*}$-algebra. Since $\left\{\Delta_{\mathcal{A}}^{i t}\right\}_{t \in \mathbb{R}}$ is a one-parameter group of $*$-automorphisms of the Tomita algebra $\mathcal{B}$, it follows that $\left\{\Delta_{\mathcal{A}}^{i t}\right\}_{t \in \mathbb{R}}$ is also a one-parameter group of $*$-automorphisms of the Hilbert quasi *-algebra $\overline{\mathcal{B}}[\|\|]$.

Finally, we consider the question of when a Hilbert space can be regarded as a standard $\mathrm{HCQ}^{*}$-algebra. By Proposition 2.4, 2.8 and [9], Theorem 13.1, we have the following: 
Theorem 2.9. Let $\mathcal{H}$ be a Hilbert space. The following statements are equivalent:

(i) $\mathcal{H}$ is a standard $H C Q^{*}$-algebra.

(ii) $\mathcal{H}$ contains a left Hilbert algebra with unit as dense subspace.

(iii) There exists a von Neumann algebra on $\mathcal{H}$ with a cyclic and separating vector.

It is worth noticing, in particular, that the implication (iii) $\Rightarrow$ (i) shows that the class of standard $H C Q^{*}$-algebras is rather rich.

\section{The structure of Strict CQ*Algebras}

In this section we study when a strict $\mathrm{CQ}^{*}$-algebra is embedded in a standard $\mathrm{HCQ}^{*}$-algebra. For that, we need a GNS-like construction for a class of positive sesquilinear forms on strict $\mathrm{CQ}^{*}$-algebras $(\overline{\mathcal{A}}[\|\|], \#,\|\| \#)$. A sesquilinear form $\varphi$ on $\overline{\mathcal{A}}[\|\|] \times \overline{\mathcal{A}}[\|\|]$ is said to be positive if $\varphi(a, a) \geq 0$ for all $a \in \overline{\mathcal{A}}[\|\|]$, and $\varphi$ is said to be faithful if $\varphi(a, a)=0, a \in \overline{\mathcal{A}}[\|\|]$, implies $a=0$. Further, we need the following notion:

Definition 3.1. Let $\left(\overline{\mathcal{A}}[\|\|], \#,\|\|_{\#}\right)$ and $\left(\overline{\mathcal{B}}\left[\|\|_{1}\right], \#_{1},\|\|_{\#_{1}}\right)$ be strict $\mathrm{CQ}^{*}$ algebras. A linear map $\Phi: \overline{\mathcal{A}}[\|\|] \rightarrow \overline{\mathcal{B}}\left[\|\|_{1}\right]$ is said to be a *-homomorphism of $\left(\overline{\mathcal{A}}[\|\|], \#,\|\|_{\#}\right)$ into $\left(\overline{\mathcal{B}}\left[\|\|_{1}\right], \#_{1},\|\|_{\#_{1}}\right)$ if (i) $\Phi$ is a $*$-homomorphism of the quasi *-algebra $\overline{\mathcal{A}}[\|\|]$ into the quasi *-algebra $\overline{\mathcal{B}}\left[\|\|_{1}\right]$, that is, $\Phi(\mathcal{A}) \subset \mathcal{B}$ and $\Phi(a)^{*}=\Phi\left(a^{*}\right), \Phi(a x)=\Phi(a) \Phi(x)$ and $\Phi(x a)=\Phi(x) \Phi(a)$ for all $a \in \overline{\mathcal{A}}[\|\|]$ and $x \in \mathcal{A}$; (ii) $\Phi\left\lceil\mathcal{A}_{\#}\right.$ is a $*$-homomorphism of the $\mathrm{C}^{*}$-algebra $\mathcal{A}_{\#}$ into the $\mathrm{C}^{*}$ algebra $\mathcal{B}_{\#_{1}}$. A bijective (resp. injective) $*$-homomorphism $\Phi$ such that $\Phi(\mathcal{A})=\mathcal{B}$ and $\Phi\left(\mathcal{A}_{\#}\right)=\mathcal{B}_{\#_{1}}$ is called a $*$-isomorphism of $\left(\overline{\mathcal{A}}[\|\|], \#,\|\|_{\#}\right)$ onto (resp. into) $\left(\overline{\mathcal{B}}\left[\|\|_{1}\right], \#_{1},\|\|_{\#_{1}}\right) . \mathrm{A} *$-homomorphism $\Phi$ is said to be contractive if $\|\Phi(a)\|_{1} \leq\|a\|$ for all $a \in \overline{\mathcal{A}}[\|\|]$. A contractive $*$-isomorphism whose inverse is also contractive is called an isometric $*$-isomorphism.

Theorem 3.2. Let $\left(\overline{\mathcal{A}}[\|\|], \#,\|\|_{\#}\right)$ be a strict $C Q^{*}$-algebra with quasi-unit u. Then the following statements are equivalent:

(i) There exists a contractive *-homomorphism (resp. *-isomorphism) of the strict $C Q^{*}$-algebra $\left(\overline{\mathcal{A}}[\|\|], \#,\|\|_{\#}\right)$ into a $H C Q^{*}$-algebra $\left(\overline{\mathcal{B}}\left[\|\|_{1}\right], \#_{1}\right)$.

(ii) There exists a (resp. faithful) positive sesquilinear form $\varphi$ on $\overline{\mathcal{A}}[\|\|] \times \overline{\mathcal{A}}[\|\|]$ satisfying

(ii) $)_{1} \varphi(x, y)=\varphi\left(u, x^{\#} y\right), \forall x, y \in \mathcal{A}$;

(ii) $)_{2}|\varphi(x, y)| \leq\|x\|\|y\|, \forall x, y \in \mathcal{A}$;

(ii) $)_{3} \varphi(x, y)=\varphi\left(y^{*}, x^{*}\right), \forall x, y \in \mathcal{A}$;

Further, $\left(\overline{\mathcal{B}}\left[\|\|_{1}\right], \#_{1}\right)$ is standard if and only if

(ii) $4 \varphi\left(x^{*}, x^{\#}\right) \geq 0, \forall x \in \mathcal{A}$.

Proof. (i) $\Rightarrow$ (ii) We put

$$
\varphi(a, b)=(\Phi(a) \mid \Phi(b)), \quad a, b \in \overline{\mathcal{A}}[\|\|],
$$

where $(\mid)$ is the inner product defined by the Hilbertian norm \|\|$_{1}$ on $\overline{\mathcal{B}}[\|\|]$. Then it is easily shown that $\varphi$ is a positive sesquilinear form on $\overline{\mathcal{A}}[\|\|] \times \overline{\mathcal{A}}[\|\|]$ satisfying the condition (ii) $)_{1} \sim(\text { ii })_{3}$. If $\left(\overline{\mathcal{B}}\left[\|\|_{1}\right], \#_{1}\right)$ is standard, then $(\text { ii })_{4}$ follows from Lemma 2.5.

(ii) $\Rightarrow$ (i) We put $\mathcal{N}_{\varphi}=\{a \in \overline{\mathcal{A}}[\|\|] ; \varphi(a, a)=0\}$. Then $\mathcal{N}_{\varphi}$ is a subspace of $\overline{\mathcal{A}}[\|\|]$ and, due to the positivity of $\varphi$, which implies $\varphi(a, b)=\overline{\varphi(b, a)}$ for each $a, b \in \overline{\mathcal{A}}[\|\|]$, 
it follows that the quotient space $\lambda_{\varphi}(\overline{\mathcal{A}}[\|\|]) \equiv \overline{\mathcal{A}}[\|\|] / \mathcal{N}_{\varphi}=\left\{\lambda_{\varphi}(a) \equiv a+\mathcal{N}_{\varphi} ; a \in\right.$ $\overline{\mathcal{A}}[\|\|]\}$ is a pre-Hilbert space with inner product $\left(\lambda_{\varphi}(a) \mid \lambda_{\varphi}(b)\right)_{\varphi}=\varphi(a, b), a, b \in$ $\overline{\mathcal{A}}[\|\|]$. We denote by \|\|$_{\varphi}$ the norm defined by the inner product $(\mid)_{\varphi}$ and by $\mathcal{H}_{\varphi}$ the completion of $\lambda_{\varphi}(\overline{\mathcal{A}}[\|\|])\left[\|\|_{\varphi}\right]$. Since $\mathcal{A}$ is \|\| -dense in $\overline{\mathcal{A}}[\|\|]$, it follows that

(ii) $)_{2}^{\prime}|\varphi(a, b)| \leq\|a\|\|b\|, \forall a, b \in \overline{\mathcal{A}}[\|\|]$;

(ii) ${ }_{3}^{\prime} \varphi(a, b)=\varphi\left(b^{*}, a^{*}\right), \forall a, b \in \overline{\mathcal{A}}[\|\|]$, and since (ii) ${ }^{\prime}$ and $\|x\| \leq\|x\|_{\#}, \forall x \in \mathcal{A}$, it follows that

(ii) ${ }_{1}^{\prime} \varphi(x, y)=\varphi\left(u, x^{\#} y\right), \forall x, y \in \mathcal{A}_{\#}$.

By (ii) ${ }_{2}^{\prime} \mathcal{A}_{\varphi} \equiv \lambda_{\varphi}(\mathcal{A})$ is a dense subspace of the Hilbert space $\mathcal{H}_{\varphi}$ and further, it is a $*$-algebra equipped with the multiplication $\lambda_{\varphi}(x) \lambda_{\varphi}(y)=L_{\lambda_{\varphi}(x)} \lambda_{\varphi}(y) \equiv \lambda_{\varphi}(x y)$ and the involution $\lambda_{\varphi}(x)^{*} \equiv \lambda_{\varphi}\left(x^{*}\right)$. By (ii) ${ }_{3}^{\prime}$ the involution $\lambda_{\varphi}(x) \rightarrow \lambda_{\varphi}(x)^{*}$ can be extended to the isometric involution $J_{\varphi}$ on $\mathcal{H}_{\varphi}$. By (ii) ${ }_{1}^{\prime}$ the linear functional on the $\mathrm{C}^{*}$-algebra $\mathcal{A}_{\#}: x \rightarrow \varphi(x, u)$ is positive, and so $\varphi\left(y^{\#}\left(x^{\#} x\right) y, u\right) \leq\|x\|_{\#}^{2} \varphi(y, y)$ for each $x, y \in \mathcal{A}$. Hence it follows from (ii) 1 that

$$
\left\|\lambda_{\varphi}(x) \lambda_{\varphi}(y)\right\|_{\varphi}^{2}=\varphi(x y, x y)=\varphi\left(y^{\#} x^{\#} x y, u\right) \leq\|x\|_{\#}^{2}\left\|\lambda_{\varphi}(y)\right\|_{\varphi}^{2}
$$

for each $x, y \in \mathcal{A}$, so that $L_{\lambda_{\varphi}(x)}$ is bounded and $\left\|L_{\lambda_{\varphi}(x)}\right\| \leq\|x\|_{\#}$ for each $x \in \mathcal{A}$. Thus $\mathcal{H}_{\varphi}=\overline{\mathcal{A}_{\varphi}}\left[\|\|_{\varphi}\right]$ is a Hilbert quasi $*$-algebra. Further, the map $\lambda_{\varphi}(x) \rightarrow$ $\lambda_{\varphi}(x)^{\#} \equiv \lambda_{\varphi}\left(x^{\#}\right)$ is an involution of $\mathcal{A}_{\varphi}$ and by (ii) ${ }_{1} L_{\lambda_{\varphi}(x)}^{*}=L_{\lambda_{\varphi}(x)} \#_{1}$ for each $x \in \mathcal{A}$. Hence, $\left(\overline{\mathcal{A}_{\varphi}}\left[\|\|_{\varphi}\right], \#_{1}\right)$ is a $\operatorname{HCQ}^{*}$-algebra. Here we put $\Phi(a)=\lambda_{\varphi}(a), a \in$ $\overline{\mathcal{A}}[\|\|]$. Then it is easily shown that $\Phi$ is a $*$-homomorphism of the strict $\mathrm{CQ}^{*}$ algebra into the $\mathrm{HCQ}^{*}$-algebra $\left(\overline{\mathcal{A}_{\varphi}}\left[\|\|_{\varphi}\right], \#_{1}\right)$ satisfying $\Phi\left(\mathcal{A}_{0}\right)=\mathcal{A}_{\varphi}$, and by (ii) ${ }_{2}^{\prime}$ it is contractive. Suppose that $\varphi$ is faithful. Then the $*$-representation of the $\mathrm{C}^{*}$-algebra $\mathcal{A}_{\#}$ on $\mathcal{H}_{\varphi}$ defined by $x \rightarrow L_{\lambda_{\varphi}(x)}, x \in \mathcal{A}_{\#}$ is faithful, which implies that $\left\|L_{\lambda_{\varphi}(x)}\right\|=\|x\|_{\#}$ for each $x \in \mathcal{A}_{\#}$. Further, since $\Phi\left(\mathcal{A}_{0}\right)=\mathcal{A}_{\varphi}$, it follows that $\Phi\left(\mathcal{A}_{\#}\right)=\left(\mathcal{A}_{\varphi}\right)_{\#_{1}}$ and $\Phi\left\lceil\mathcal{A}_{\#}\right.$ is a $*$-isormorphism of the $\mathrm{C}^{*}$-algebra $\mathcal{A}_{\#}$ onto the $\mathrm{C}^{*}$ algebra $\left(\mathcal{A}_{\varphi}\right)_{\#_{1}}$. Hence $\Phi$ is a $*$-isomorphism of $\left(\overline{\mathcal{A}}[\|\|]\right.$, \#, \|\|$\left._{\#}\right)$ into $\left(\overline{\mathcal{A}_{\varphi}}\left[\|\|_{\varphi}\right]\right.$, \#1 $)$. By Lemma 2.5 , the $\mathrm{HCQ}^{*}$-algebra $\left(\overline{\mathcal{A}_{\varphi}}\left[\|\|_{\varphi}\right], \#_{1}\right)$ is standard if and only if (ii) $)_{4}$ holds. This completes the proof.

Now the question arises as to whether positive sesquilinear forms as described in (ii) do really exist. The answer is certainly positive due to the existence of standard $\mathrm{HCQ}^{*}$-algebras stated in Theorem 2.9. Indeed, the inner product $\langle$,$\rangle of a$ left Hilbert algebra satisfies conditions (ii) $)_{1}-(\mathrm{ii})_{4}$.

Furthermore, Theorem 3.2 answers the main question in this section: any form $\varphi$ over a strict $\mathrm{CQ}^{*}$-algebra $\left(\overline{\mathcal{A}}[\|\|], \#,\|\|_{\#}\right)$ with quasi-unit, can be used to construct a $\mathrm{HCQ}^{*}$-algebra where $\overline{\mathcal{A}}$ is contractively embedded.

\section{ACKNOWLEDGMENT}

We acknowledge the financial support of the Gruppo Nazionale per l'Analisi Funzionale e le Applicazioni del C.N.R. and of the Italian Ministry of Scientific Research, and of the Japan Private School Promotion Foundation.

\section{REFERENCES}

1. F.Bagarello and C.Trapani, States and representations of $C Q^{*}$-algebras, Ann. Inst. H. Poincaré 61, 103-133 (1994) MR 95j:46062

2. F.Bagarello and C.Trapani, CQ*-algebras: structure properties, Publ. Res. Inst. Math. Sci., Kyoto Univ. 32, 85-116 (1996) MR 97d:46064 
3. F.Bagarello and C.Trapani, $L^{p}$-spaces as quasi ${ }^{*}$-algebras, J. Math. Anal. Appl . 197, 810-824 (1996) MR 96k:46064

4. F.Bagarello and C.Trapani, The Heisenberg dynamics of spin systems:a quasi *-algebras approach, J. Math. Phys. 37, 4219-4234 (1996) MR 97j:82005

5. I.M.Gelfand and N.Ya.Vilenkin, Generalized functions Vol. 4, Academic Press, New York and London, 1964 MR 55:8786d

6. G.Lassner, Algebras of unbounded operators and quantum dynamics, Physica, 124A, 471-479 (1984) CMP 17:01

7. G.Lassner and G.A.Lassner, Qu*-algebras and twisted product, Publ. Res. Inst. Math. Sci., Kyoto Univ. 25, 279-299 (1989) MR 90h:47087

8. S.Stratila and L.Szido, Lectures on von Neumann Algebras, Revision of 1975 original, Abacus Press, Tunbridge Wells, 1979 MR 81j:46089

9. M.Takesaki, Tomita's theory of modular Hilbert algebras and its applications, Lecture Notes in Mathematics, 128 Springer-Verlag, 1970 MR 42:5061

10. C.Trapani, Quasi *-algebras of operators and their applications, Rev. Math. Phys. 7 13031332 (1995) MR 97a:47070

11. A.Van Daele, A new approach to the Tomita-Takesaki theory of generalized Hilbert algebras, J. Functional Analysis 15, 378-393 (1974) MR 49:11264

Dipartimento di Matematica, Università di Palermo, I-90128 Palermo, Italy

E-mail address: bagarello@www.unipa.it

Department of Applied Mathematics, Fukuoka University, J-814-80 Fukuoka, Japan

E-mail address: a-inoue@fukuoka-u.ac.jp

Dipartimento di Scienze Fisiche ed Astronomiche, Università di Palermo, I-90123 PALERmo, Italy

E-mail address: trapani@unipa.it 